\title{
Metodologías para analizar los factores que limitan al docente en la integración inclusiva de los niños y niñas con necesidades educativas especiales
}

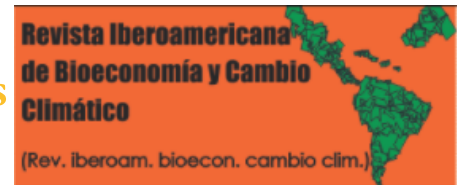

\author{
Methodologies to analyze the factors that limit the teacher in \\ the Inclusive integration of children with educational needs \\ special
}

\author{
Lindo, Ivett Francisca; Zúniga-González, Carlos Alberto; Editor \\ Academico Prof. Angel Sol-Sanchez
}

\section{Ivett Francisca Lindo}

ivett.lindo@post.unanleon.edu.ni

Ministerio de Educación (MINED)-Somotillo.,

Nicaragua

Carlos Alberto Zúniga-González

czuniga@ct.unanleon.edu.ni

Universidad Nacional Autónoma de Nicaragua, León.,

Nicaragua

Editor Academico Prof. Angel Sol-Sanchez

sol@colpos.mx

Colegio de postgraduados, México

Revista Iberoamericana de Bioeconomía y Cambio Climático

Universidad Nacional Autónoma de Nicaragua, León, Nicaragua ISSN-e: 2410-7980

Periodicidad: Semestral

vol. 3, núm. 5, 2017

czuniga@ev.unanleon.edu.ni

Recepción: 12 Junio 2017

Aprobación: 26 Julio 2017

URL: http://portal.amelica.org/ameli/

jatsRepo/394/3941752005/index.html

DOI: https://doi.org/10.5377/ribcc.v3i5.5942
Resumen: Este estudio pretende analizar las diferentes estrategias metodológicas aplicadas por los y las docentes a niños con necesidades educativas asociadas a una discapacidad las técnicas utilizadas para la recopilación de la información fueron: Análisis documental entrevistas observación a .Director, Docentes Padres de Familias, especialistas en Educación inclusiva y a niños y niñas con necesidades educativas especiales. Actualmente en la sociedad en que vivimos surgen diversas necesidades educativas las cuales se presentan por diversas razones formas y causas, transformándose en impedimentos para el aprendizaje de los niños (as) por lo que requieren de una mayor atención para superar esos obstáculos (deficiencias). Por lo tanto cabe mencionar que el centro educativo en donde se realizará la investigación apunta a una educación inclusiva donde se atienden a niños con necesidades educativas especiales a una discapacidad.

Palabras clave: Educación inclusiva, Estrategias metodológicas integración, Enfoque inclusivo.

Abstract: This study aims to analyze the different methodological strategies appliedby teachers to children with educational needs associated with aDisability The techniques used for the collection of information were:Documentary analysis interviews observation to .Director, Teachers Parents ofFamilies, specialists in inclusive education and children with needsspecial educational Currently in the society in which we live, diverse needs ariseeducational which are presented for various reasons, forms and causes,becoming impediments to children's learning so thatthey require more attention to overcome these obstacles (deficiencies). Therefore it is worth mentioning that the educational center where theresearch aims at an inclusive education where children are cared forspecial educational needs to a disability.

Keywords: Inclusive education, Methodological strategies integration, Inclusive approach. 


\section{INTRODUCCIÓN}

La ley general de educación, la convención de los derechos del niño, el código de la niñez y la adolescencia, la ley763 "Ley de los derechos de las personas con discapacidad", entre otros asume la tarea de construir un nuevo modelo educativo basado en la transformación de la conciencia y la restitución de los derechos, teniendo como centro el desarrollo humano y como fin el bienestar social de los niños y niñas Nicaragüense (Herrera, 2004; Miguelez, 2000; Mackernan, 1999).

La diversidad de funciones del profesor, ahora también, como acompañante y guía, hacen poco claras sus funciones para las instituciones que contratan. Por eso es necesario que exista un reconocimiento de que la función del profesor ha variado y ya no sólo es necesario el título profesional como docente hace falta una formación especializada, de la misma forma que se le exige a lo demás profesionales, debemos considerar que la forma de enseñanza no es estática, evoluciona constantemente y el docente es el que determina esos cambios en su protagonismo docente (Herrera, 2004).

Necesitamos formar didácticamente o pedagógicamente al profesor, aportar las condiciones especiales y apropiadas para la labor docente.

En cuanto a los docentes, es interesante mencionar que estos constituyen un factor de suma importancia para atender a la diversidad de alumnos con Necesidades Educativas Especiales, como lo señala Marchessi (1999), el docente en el aula será el principal recurso para la instrucción de los alumnos que presenten algún problema de aprendizaje. Esto conlleva un esfuerzo permanente por mejorar sus competencias profesionales y desarrollar sus habilidades didácticas.

Lo antes expuesto, se concretiza en el plan estratégico de educación en el cual se establece como meta contribuir una educación para todos y todas con calidad, justicia y eficacia que permita el desarrollo pleno de los niños y niñas incluyendo a las personas con necesidades educativas asociadas o no a discapacidad y es por eso que se implementarán diferentes estrategias metodológicas con recursos adaptados para garantizar que los niños y niñas con necesidades educativas asociadas o no a discapacidad accedan, permanezcan y promuevan en los diferentes niveles educativos y modalidades para que el proceso de aprendizaje sea más eficaz y pertinente (Becerra,et.al., 2012; Denegri, 2015; ; Rodriguez, 2011).

\section{DESCRIPCIÓN DE INVESTIGACIÓN ACCIÓN.}

Beltran, (2003), manifiesta que la investigación acción se puede considerar como un término genérico que hace referencia a una amplia gama de estrategias realizadas para mejorar el sistema educativo y social.

Por otro lado la investigación- Acción

El término investigación-acción fue propuesto por primera vez en 1944 por el psicólogo social Kurt Lewin (1946) y fue desarrollado por Lawrence Stenhouse, Gary Anderson, Carr y Stephen Kemmis (Gillard, et. al 1984). Esta forma de indagación fue puesta a prueba en diversos contextos como la igualación de oportunidades para obtener empleo, la causa y curación de perjuicios en los niños, la socialización de las bandas callejeras y el mejoramiento en la formación de jóvenes líderes en la Norteamérica de posguerra.

Existen diversas definiciones de investigación-acción; las líneas que siguen recogen algunas de ellas.

Elliott (1991), el principal representante de la investigación-acción desde un enfoque interpretativo define la investigación-acción en 1993 como «un estudio de una situación social con el fin de mejorar la calidad de la acción dentro de la misma». La entiende como una reflexión sobre las acciones humanas y las situaciones sociales vividas por el profesorado que tiene como objetivo ampliar la comprensión (diagnóstico) de los docentes de sus problemas prácticos. Las acciones van encaminadas a modificar la situación una vez que se logre una comprensión más profunda de los problemas.

Elliott (1993) define la investigación-acción como «un estudio de una situación social con el fin de mejorar la calidad de la acción dentro de la misma». La entiende como una reflexión sobre las acciones humanas y las 
situaciones sociales vividas por el profesorado que tiene como objetivo ampliar la comprensión (diagnóstico) de los docentes de sus problemas prácticos. Las acciones van encaminadas a modificar la situación una vez que se logre una comprensión más profunda de los problemas.

Con Gillard, et. al (1984) la investigación-acción no sólo se constituye como ciencia práctica y moral, sino también como ciencia crítica. Para este autor la investigación acción es: una forma de indagación autor reflexiva realizado por quienes participan (profesorado, alumnado, o dirección por ejemplo) en las situaciones sociales (incluyendo las educativas) para mejorar la racionalidad y la justicia de: a) sus propias prácticas sociales o educativas; b) su comprensión sobre las mismos; y c) las situaciones e instituciones en que estas prácticas se realizan (aulas o escuelas, por ejemplo).

\section{Características de la investigación acción.}

1- Se trata de una forma de investigación para enlazar el enfoque experimental de la ciencia social con programas de acción social que respondan a los problemas sociales principales.

2- La investigación-acción inicia el cuestionamiento del fenómeno desde lo habitual, transitando sistemáticamente, hasta lo filosófico.

3- Mediante la investigación-acción se pretende tratar de forma simultánea conocimientos y cambios sociales, de manera que se unan la teoría y la práctica.

4- Es participativa. Las personas trabajan con la intención de mejorar sus propias prácticas.

5- La investigación sigue una espiral introspectiva: una espiral de ciclos de planificación, acción, observación y reflexión.

6- Es colaborativa, se realiza en grupo por las personas implicadas.

7- Induce a teorizar sobre la práctica.

En esencia, Kurt Lewin (1946) sugería que las tres características más importantes de la investigación acción moderna eran:

- su carácter participativo,

- su impulso democrático y

- su contribución simultánea al conocimiento en las ciencias sociales.

Las tres modalidades de investigación-acción (Carr y Kemmis 1986)

1- Técnica: Su Objetivo es la efectividad, eficiencia de la práctica educativa. Desarrollo profesional.

2- Practica: La comprensión de los prácticos. La transformación de su conciencia.

3- Emancipadora: Emancipación de los participantes de los dictados de la tradición, auto decepción, corrección. Su crítica de la sistematización burocrática. Transformación de la organización y del sistema educativo.

\section{Modelos del proceso de investigación-acción}

\section{Modelo de Lewin}

Lewin (1946) describió la investigación-acción como ciclos de acción reflexiva. Cada ciclo se compone de una serie de pasos: planificación, acción y evaluación de la acción. Comienza con una «idea general» sobre un tema de interés sobre el que se elabora un plan de acción. Se hace un reconocimiento del plan, sus posibilidades y limitaciones, se lleva a cabo el primer paso de acción y se evalúa su resultado. El plan general es revisado a la luz de la información y se planifica el segundo paso de acción sobre la base del primero.

\section{Modelo de Kemmís}

Kemmis (1989), apoyándose en el modelo de Lewin, elabora un modelo para aplicarlo a la enseñanza. El proceso lo organiza sobre dos ejes: uno estratégico, constituido por la acción y la reflexión; y otro organizativo, 
constituido por la planificación y la observación. Ambas dimensiones están en continua interacción, de manera que se establece una dinámica que contribuye a resolver los problemas y a comprender las prácticas que tienen lugar en la vida cotidiana de la escuela.

El proceso está integrado por cuatro fases o momentos interrelacionadas: planificación, acción, observación y reflexión.

\section{Modelo de Elliott}

El modelo de Elliott (1991), toma como punto de partida el modelo cíclico de Lewin, que comprendía tres momentos: elaborar un plan, ponerlo en marcha y evaluarlo; rectificar el plan, ponerlo en marcha y evaluarlo, y así sucesivamente.

En el modelo de Elliott aparecen las siguientes fases:

Identificación de una idea general. Descripción e interpretación del problema que hay que investigar.

Exploración o planteamiento de las hipótesis de acción como acciones que hay que realizar para cambiar la práctica.

Construcción del plan de acción. Es el primer paso de la acción que abarca: la revisión del problema inicial y las acciones concretas requeridas; la visión de los medios para empezar la acción siguiente, y la planificación de los instrumentos para tener acceso a la información. Hay que prestar atención a:

- La puesta en marcha del primer pasó en la acción.

- La evaluación.

- La revisión del plan general.

\section{Modelo de Whitehead}

Whitehead (1989), crítico con las propuestas de Kemmis y de Elliott, por entender que se alejan bastante de la realidad educativa convirtiéndose más en un ejercicio académico que en un modelo que permita mejorar la relación entre teoría educativa y autodesarrollo profesional.

\section{Enfoques investigativos.}

El enfoque es la perspectiva o el horizonte de sentido desde el que se observa la realidad. Por lo tanto en éste cuentan los intereses, las intencionalidades y los conocimientos con los que el investigador percibe, categoriza y conceptualiza los fenómenos estudiados Martínez (2006).

\section{Tipo de Investigación}

Se realizara una investigación acción participativa con enfoque cualitativo que permitirá conocer la esencia de la realidad social en su propio marco natural, la realidad del fenómeno o problema a estudiar se consideran todos los factores que puedan ejercer influencias en el objeto de estudio.

La muestra estará conformada por una: directora del centro educativo

3 docentes 10 estudiantes con necesidades educativas y 5 niños con necesidades comunes.

Las técnicas seleccionadas para recopilar la información serán: la observación, entrevista, así como la revisión de documentos Análisis intensivo de la información y uso de técnicas de triangulación.

En este primer propósito y durante el proceso de investigación consulte a docente, padres de familias para reconocer las características generales de niños (as) con necesidades educativas especiales y sus dificultades y encontré lo siguiente: retraso mental leve, dificultades en el habla problemas psicomotor síndrome Down .El cual implementare estrategias metodológicas adecuadas para su desarrollo.

Entre las estrategias pedagógicas aplicadas en el aula de clase a los niños y niñas con necesidades educativas serán: trabajos grupales, uso de sistema de Braille, láminas educativas, atención directa e individualizada a cada niño (a).Cabe mencionar que si queremos desarrollarnos en la educación inclusiva el currículo escolar 
tiene que proporcionar oportunidades adecuándolo para que todos los niños y niñas logren los aprendizajes y desarrollen al máximo sus conocimientos y capacidades.

Elementos que obstaculizan la atención a niños (as) con necesidades educativas asociadas a una discapacidad plantean tanto los maestros como los facilitadores y padres de familias que los principales problemas que inciden en la atención de los niños son la falta de materiales didácticos, concreto, libros de textos adecuados falta de capacitación en el lenguaje de señas y adecuaciones curriculares entre otros .Como se puede constatar son muchas las dificultades que obstaculizan el proceso de enseñanza aprendizaje por lo tanto será un trabajo arduo pero enriquecedor para mí (Gonzales, et.al., 1999).

\section{Conclusión.}

Como producto de este trabajo, discusión, triangulación de la información, análisis y reflexión se puede concluir que este estudio es de gran importancia, porque permitirá adquirir conocimientos referidos a las estrategias metodológicas que se aplican en las aulas de clases para la formación académica de los niños(as) que presentan necesidades educativas especiales, asociadas a una discapacidad sobre el aspecto de la inclusividad educativa, lo cual conllevará a mejorar el proceso de enseñanza aprendizaje como propósito principal de la Institución que rige la educación en Nicaragua (Bisquerra, 2009).

Algunos docentes manejan estrategias de enseñanza para el aprendizaje, pero son estrategias propias de la educación regular del programa y el currículo de la educación primaria ya que no hay un programa específico para atender la inclusividad. Por lo tanto se hace necesario que los docentes sean capacitados en estrategias específicas para atender a la diversidad, como es el caso de uso adecuado de los diferentes materiales y métodos para distintas necesidades educativas asociadas a una discapacidad.

\section{REFERENCIAS}

Herreras, E. B. (2004). La docencia a través de la investigación-acción. Revista iberoamericana de educación, 35(1), 1-9. BISQUERRA ALZINA, R. (2009). Metodología de la investigación educativa. En A. LATORRE BELTRAN, La investigación acción (370-394). Madrid: La Muralla.

Becerra Labra, C., Gras-Martí, A., Hernández Hernández, C., Montoya Vargas, J., Osorio Gómez, L. A., \& Sancho Vinuesa, T. (2012). Renewal of University Education based on Proofs (Renovación de la Enseñanza Universitaria Basada en Evidencias REUBE): A methodology of flexibla action. Perfiles educativos, 34(135), 62-77.

Denegri, A., Aguirre, A., \& Salinas, V. P. (2015). Entramando vínculos con niñas, niños y adolescentes y con redes comunitarias. In V Congreso Internacional de Investigación de la Facultad de Psicología de la Universidad Nacional de La Plata (La Plata, 2015).

Beltran, A. L. (2003). La investigación-acción: Conocer y cambiar la práctica educativa (Vol. 179). Grao.

Elliot, J. (1993). El cambio educativo desde la investigación-acción, Madrid: Morata.

González, JP, Ullastres, Á. M., y Carretero, M. (1999). Psicología Evolutiva. 2. Desarrollo cognitivo y sociales del niño. Alianza editorial.

Herreras, E. B. (2004). La docencia a través de la investigación-acción. Revista iberoamericana de educación, 35(1), 1-9.

Lewin, K. (1946). Action research and minority problems. Journal of social issues, 2(4), 34-46.

Miguélez, M. M. (2000). La investigación-acción en el aula. Agenda académica, 7(1), 27.

McKernan, J. (1999). Investigación-acción y curriculum: métodos y recursos para profesionales reflexivos. Ediciones Morata.

Rodríguez, J. M. (2011). Métodos de investigación cualitativa. Revista Silogismo, 4(8), 11-11. 
Ivett Francisca Lindo, et al. Metodologías para analizar los factores Que limitan al docente en la...

Elliott, J. (1991). Estudio del curriculum escolar a través de la investigación interna. Revista Interuniversitaria de Formación del Profesorado, 10, 45-68

Gillard, G., Kemmis, S., \& Bartlett, L. (1984). Collaboration between two universities in course development. Higher Education Research and Development, 3(1), 71-79.

Kemmis, S. (1989). Improving schools and teaching through educational action research. Singapore journal of education, 6-30.

Martínez, M. (2006). La investigación cualitativa (síntesis conceptual). Revista IIPSI. Facultad de Psicologia. Vol 9 (1) pp123-146.

Whitehead, J. (1989). Creating a living educational theory from questions of the kind,'How do I improve my practice?'. Cambridge journal of Education, 19(1), 41-52. 